\title{
Xitris: a software to acquire, display, compress, and publish data in real time using distributed mode for the Solar Radio Interferometer RIS
}

\author{
Victor De la Luz ${ }^{1}$ and Alejandro Lara ${ }^{2}$ \\ Manuscript received on January 29, 2013 / accepted on April 10, 2013
}

\begin{abstract}
Upgrading the infrastructure of old scientific instruments requires the development of new hardware and software which may be expensive (in general, these projects lack of enough resources to acquire fast and modern infrastructure to become competitive and functional in the era of digital data). Particularly, the development of software for data acquisition in real time is one of the most important topics in experimental science and industry. However, the constant improvements on the data acquisition hardware, discourages the development of software highly optimized in order to minimize the consumption of resources like processor time and read/write memory, etc. In this work, we present a Open Source code called "X Interface to RIS" (Xitris). This is a modular and distributed code which, using relatively slow processors and low memory hardware, is able to acquire, display, compress and publish (via Internet) digital data in real time. Xitris was developed for the Solar Radio Interferometer (RIS) at Geophysics Institute of the National University of Mexico (UNAM) but nowadays is working in another 3 radio observatories.
\end{abstract}

Keywords: distributive algorithms, analog/digital converter.

\section{INTRODUCTION}

The Analog/Digital Converters (ADC) translate analog electrical signals that represents physical parameters (for example pressure, light, temperature) in digital data, represented in binary code. An ADC has four main characteristics: the velocity of the conversion $(\mathrm{Hz})$, the resolution of the data (bits), the noise of the conversion (mV), and the range of conversion (V). The ADC hardware delivers the data sequentially. The process control, e.g. acquisition rate, error control and storage, is controlled by software. Although nowadays, to make ADCs boards is relatively cheap, the challenge remains in the software development.

The Laboratory of Solar Physics in the Institute of Geophysics of the National University of Mexico is responsible of the Solar Radio Interferometer (RIS, Fig. 1). This instrument was donated by the former Soviet Union 30 years ago. At the beginning, the RIS was operated at the National Institute of Astrophysics (INAOE) in the state of Puebla, Mexico, and few years later the RIS was moved to its actual location in Mexico City.

The RIS has been updated several times in the past 30 years, unfortunately, most of the documentation was lost or are in Russian language and obsolete, the available funds for the project are very limited and the computer infrastructure at the time when we started the XITRIS project was old. At that time, the laboratory bought a comercial Analog/Digital Converter (ADC) to improve the record of the RIS data.

Correspondence to: Victor De la Luz - E-mail: vdelaluz@geofisica.unam.mx

${ }^{1}$ SCiESMEX, Instituto de Geofisica, Unidad Michoacan, Universidad Nacional Autonoma de Mexico, Morelia, CP. 58190 Michoacan, Mexico.

${ }^{2}$ Instituto de Geofísica, Universidad Nacional Autónoma de México, D.F., Mexico. 


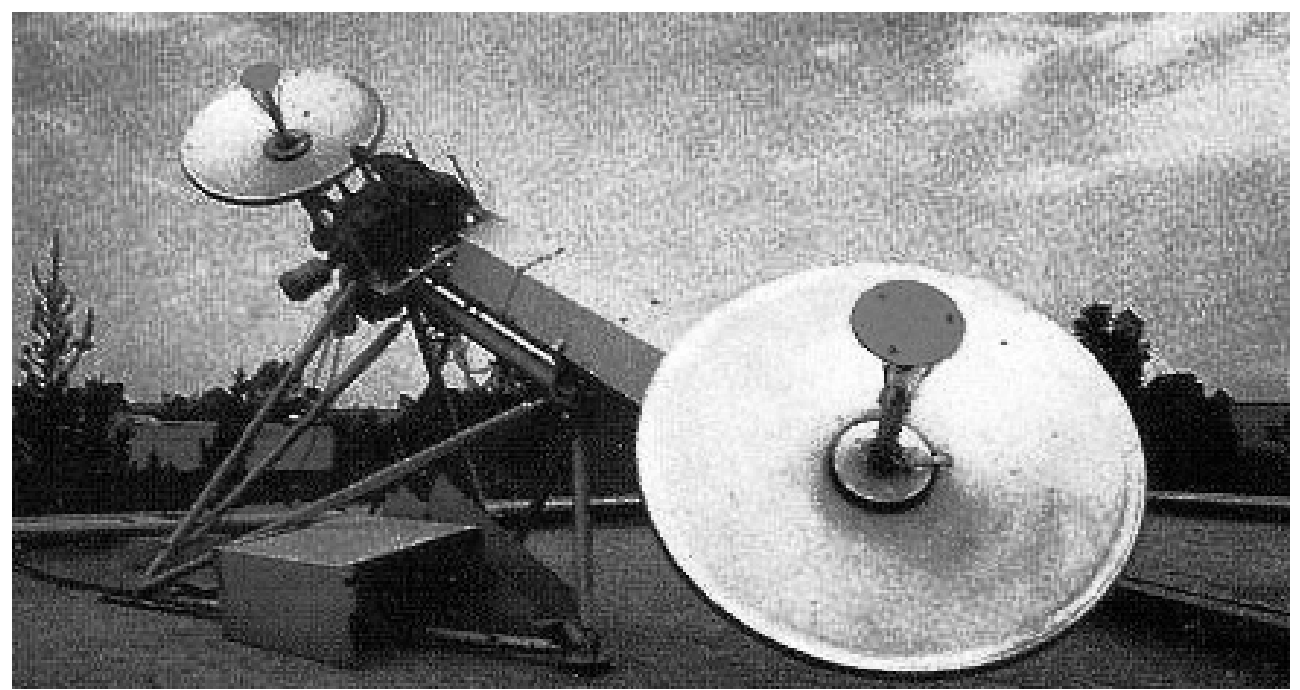

Figure 1 - The Solar Radio Interferometer (RIS) in its final location at the National University in Mexico, in Mexico City.

The acquisition of a new ADC board implied the necessity of either buying or developing new software. The former option was avoided due to the limited budget and due to the fact that the laboratory is focused in the development of technology.

The attempt to create a new acquisition software using LabVIEW package failed [8]. An analysis showed that the old hardware infrastructure, with monolithic design, prevented the running of the ADC acquisition software [7]. Therefore, the design of a new distributed software was necessary.

In this work, we present a general purpose algorithm intended to acquire, display, compress, and publish data in real time and in a distributed mode. The "X Interface to RIS" (Xitris) code can be distributed in four computers in order to maximize the use of resources, and may be used in any observatory with high rate of data acquisition. The software engineering process was carried out using eXtreme Programming (XP).

The paper is organized as follows: In Section 2 we present the status of the old Recorder System and the infrastructure of the Laboratory at the time of the project starting, respectively. The algorithm and its implementation is presented in Section 3. In Section 4 we show the implementation results and finally, in Section 5 are our conclusions.

\section{THE SOLAR RADIO INTERFEROMETER (RIS)}

The old recorder system of the RIS had four chart recorders which printed a stroke in continuous paper representing four characteristics (total and polarized flux as well as two channels of interferometric signal) of the solar flux at $7 \mathrm{GHz}$ observed and processed by the RIS (Figure 2, shows an example of the total flux). This kind of plots of (counts versus time) is the main piece of work for the researchers interested in solar activity.

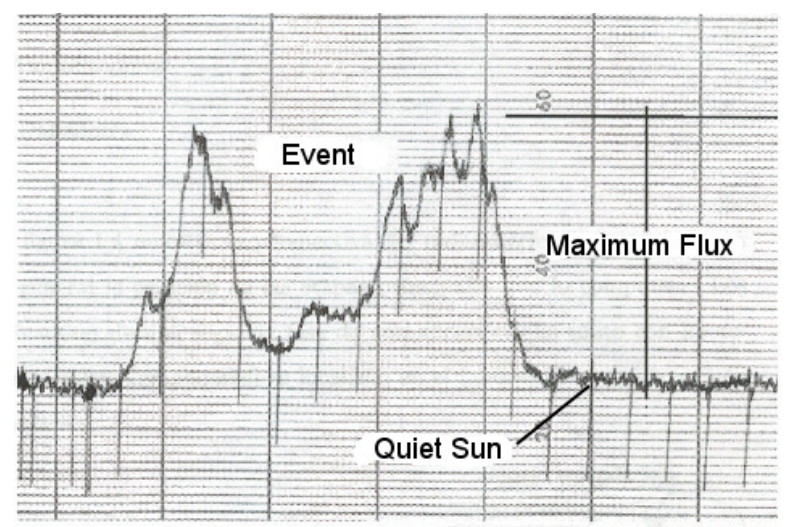

Figure 2 - One example of a solar flare observed in the total flux channel and recorded in continuous paper using the old recording system of the Solar Radio Interferometer.

The telescope started manually by the technical staff, they turned on the solar radiotelescope every day around the sunrise and marked (in the same paper of the signal) two reference levels, one with no source (zero) at the input and other form a known noise source. Finally, the radiotelescope was manually pointed to the Sun position, and the tracking system was started when the signal of the total flux channel reached its maximum level.

Once the tracking system was working, a regular line at a constant level was printed, marking the quiet sun signal. An abrupt increase of the signal may be caused by a solar event or artificial interference. 
At the beginning of the project, the laboratory had the follow infrastructure:

1. Network

- 2 IPs homologous address.

2. Hardware

- 3 Computers Pentium MMX at $166 \mathrm{MHz}$ with $46 \mathrm{Mb}$ RAM memory, with Bus Master at $66 \mathrm{MHz}$ and video card Cirrus Logic GD 5430.

- Computer 80486 with ISA interface.

- Laser Printer.

- Digital converter board ADC LabPC+ with ISA interface.

3. Software

- Debian GNU/Linux.

- Windows 95.

\section{Documentation}

- Lab-PC+ User Manual.

The main problem was the old infrastructure and the limited resources for the project. At the early stage of the project the group focused in a specific purpose software intended to solve the problem of the old RIS infrastructure. However, we realize that more observatories and/or laboratories may be in a similar situation than the RIS (in terms of software requirements) and therefore, we decided to build a general propose adquisition data software using the RIS has a study case.

\section{THE X INTERFACE TO THE RIS}

The $X$ Interface to the RIS (Xitris) is written in C using GTK+ and GDK libraries [5], except the server web-pages, for these cases we use Java [JSPs technology see Section 4]. The code runs over GNU/Linux systems. We compile the code with GCC compiler. We use eXtreme Programming (XP) as software development methodology [1]. The design includes the client-server paradigm, distributed systems and modular object-oriented technique (OOP) but using $C$ language. It is interesting to note that although the $\mathrm{C}$ language is not $00 \mathrm{P}$ (like $\mathrm{C}++$ or Java) we can use the $C$ native structures to emulate the $00 \mathrm{P}$ in a very efficient way in order to encapsulate the functionality of each piece of software and build a modular system that can be replace each library easily [9].
In our case, the most important library is related with the ADC control board. We developed generic methods which are able to be reprogrammed in order to include new ADC boards.

In eXtreme Programming there are several (short) cycles of development. The scope of each cycle is focused in: writing a short version, using simple design, testing, re-factoring, and pair programming. In this way, the process of building the software is fast and dynamic.

Prior of the starting of each cycle, it is necessary to carry out several interviews with the users in order to determine the scope of the next step of development.

The XP cycle have 4 steps: Analysis, Design, Implementation, and Tests. It is necessary analyze each step of development, design the solution, implement it, and finally test the code.

The XITRIS software has 14 cycles of development:

1. Development of the Client-Server algorithm.

2. Integration of the Server with the LabPC+.

3. Development of the graphic interface.

4. Implementation of Start/End data acquisition.

5. Data plotting.

6. Saving data.

7. Database structure.

8. Saving the status of XITRIS.

9. Implementation of tools to change of Amplification and Frequency of the data acquisition.

10. Publishing the data in Internet in Real Time.

11. Plotting the data in Real Time.

12. Implementation of the data display.

13. Detecting Solar Events.

14. Creation of install packages.

The final design includes 4 general modules (see Fig. 3):

1. The xitris-server: acquires data from an ADC board and send it trough the network. This server also configures the ADC board.

2. The xitris-client: receives the data from the xitris server, and saves and displays it the data in real time.

3. The xitris-www: plots and publishes the data on the internet in real time. This module also shows an interface of the database on the internet and produces dynamically the web-pages with the data plots in real time.

4. The xitris-analytic: is intended to display, log, and analyze the data in real time. 


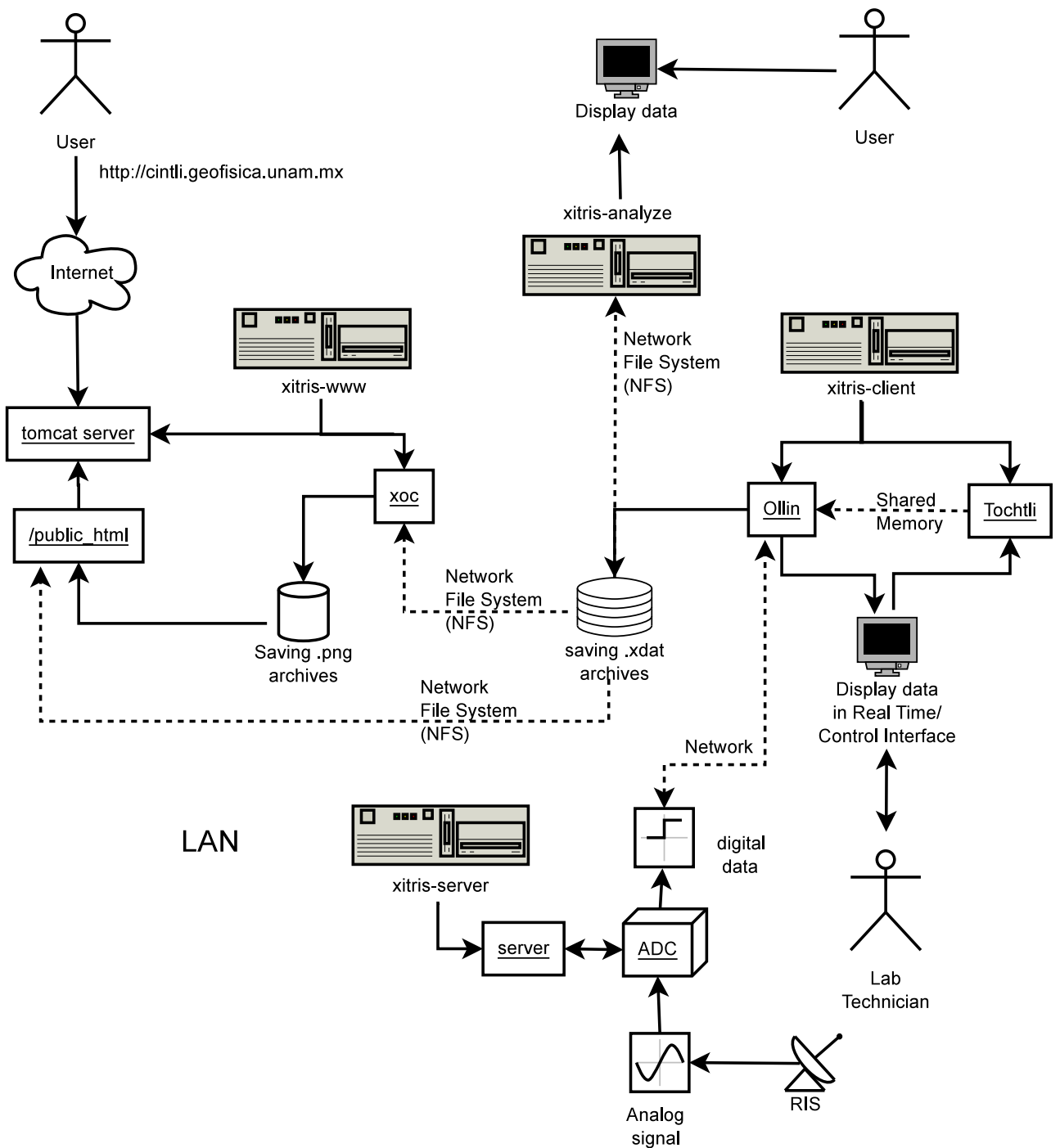

Figure 3 - Work-flow model for the Xitris project. The xitris-server collect the data and send the information by network. The Xitris-client saves and display the data. Xitris-www publish and display the data in the Internet. Xitris-analyze shows the data to other people (researchers) inside the LAN.

The xitris modules can be installed in a single or multiple computer environment. We design this 4 pieces of software in order to maximize the computer infrastructure of the RIS laboratory.

In the following sub sections we will describes each module and their interactions between them.

\section{1 xitris-server}

The xitris-server is a single piece of software that listen the calls from the xitris-clients [in the paradigm of client-server the xitrisserver module play the role of server 6$]$. The remote call includes the configuration of the ADC board (sampling frequency and amplification), the starting and the ending of the acquisi- tion process, and the error handling. For the case of the Lab$\mathrm{PC}+$, we can handle up to 4 channels simultaneously with 12 bits of precision.

The server publish a port and wait for a client request. Then, negotiate the data transmission: First, the server receives 20 integers (with the configuration from the client), configures the ADC, starts the data acquisition and sends, throughout the network socket, 4 integers each time (for each channel), if an error is detected in the ADC, the algorithm sends a signal to the client reporting that an error was detected, the client handles the error, if a change of configuration is requested, the acquisition is stopped, the $A D C$ is reconfigured and then the acquisition restarts. 
The server has modular architecture, the ADC library can be reprogrammed in order to conserve the structure and include a new ADC board. For the case of the RIS, we implement the Lab$\mathrm{PC}+$ library.

The server reads the 12 bits of data from the ADC and transform it to A2 complement integer of 32 bits (see Appendix A.1). This implementation is very fast and give us the possibility of read data in the 4 channels in milliseconds with a very old computer.

The xitris-server was installed in a old 486 PC with the ADC board. We tested the acquisition time (without graphics or data saving) and the acquisition period reached one millisecond, equivalent to the highest frequency of acquisition of the $\mathrm{Lab}-\mathrm{PC}+$, without any problem.

\section{2 xitris-client}

The xitris-client is the graphical interface between the user and the xitris-server (is the client in the client-server model). We use $\mathrm{C}$ with GTK+ and GDK libraries to build the interfaces. The ADC board can be configured remotely using this interface. The xitrisclient opens a socket to communicate with the server, sends the $A D C$ board configuration and receives the data from the server. The ADC board can be re-configured in real time. The client also saves and compress the raw data in the ".xdat" format using a sophisticated buffer that saves the data on the disk only when a threshold value is reached. This buffer is very efficient and provides a fast implementation of the saving data process in real time, using limited resources.

The .xdat format takes advantage of the 12 bits length of the ADC data words, saving 4 measurements in a single integer variable of 32 bits. The buffer grows in the memory dynamically. When the buffer is full, the data is saved on the hard disk. A converter between .xdat and ASCII was also programmed. The .xdat is saved in the directory /yyyy/mm/dd where yyyy is the year, $\mathrm{mm}$ the month and dd the day when the data was acquired.

The xitris-clients have two sub programs: "ollin" and "tochtli". Ollin is involved in the following processes: communicating with the server, displaying the data, and populate the buffer in real time. Tochtli is the interface with the user that controls Ollin software. The intercommunication between Ollin and Tochtli use an external variable via the memory. We use 36 register of 32 bits to intercommunicate the configuration from the user to the Ollin (see Appendix A.2).

The buffer library is in the Ollin source code, while the procedures to create the graphics and the intercommunication with the server are in the ImageEngine library of the Ollin source code.

\section{3 xitris-www}

This module is responsible of plot and display the data on the internet in real time. This software has two components: The real time plotter $(x \circ c)$ and the infrastructure for the website (public_html). Both codes work together in order to publish the plots and the raw data in real time on the web in the following way:

1. First, read the files generated by xitris-client (the .xdat files generated by Ollin and the .png files generated by xoc).

2. If the size of the .xdat archive changes, then it reads again the file and generates the plot (making a .png file in a public path for the internet).

3. Finally, the code dynamically generate a web-page to display the plot in real time.

The webpage of the database is generated in this module. We includes the archives of each day of observations. The web site was tested with Apache tomcat 5 and 6 . The source code is under the directory /public_html. The Xoc code was written in C and works as a daemon of the system. Another web-pages can be included to introduce information about the archives and the instrument.

\section{4 xitris-analyze}

This module can display the information saved by xitris-client, the plots are almost in real time, the time shift depends of the size of the buffer of xitris-client. It is possible to program algorithms to look for specific features over the signal using a dedicated computer. We developed this module as log of xitris system. Each event or note can be saved in this interface, the date and time is saved in the same buffer and can be recovered for further analysis.

\section{RESULTS}

The source files of the four modules are packaged and can be downloaded from [2].

In Figures 4, 5, 6, and 7 we shows the interfaces of xitris that are running in the laboratory. The Figure 4 shows the plots that xitris publish in real time in internet via xitris-www, this plot is for full day of observations but is updated each 5 minutes. The Figure 5 shows the four channel plot that we running in the laboratory to observe the raw data of the signal in real time, the plot is updated in real time. The Figure 6 is the interface to configure the amplification and the clock sampling of the LabPC+ ADC board. The Figure 7 shows the log of the observation, in this interface we make annotations over the signal about problems or remarks due to external factors that could affect the observations. 


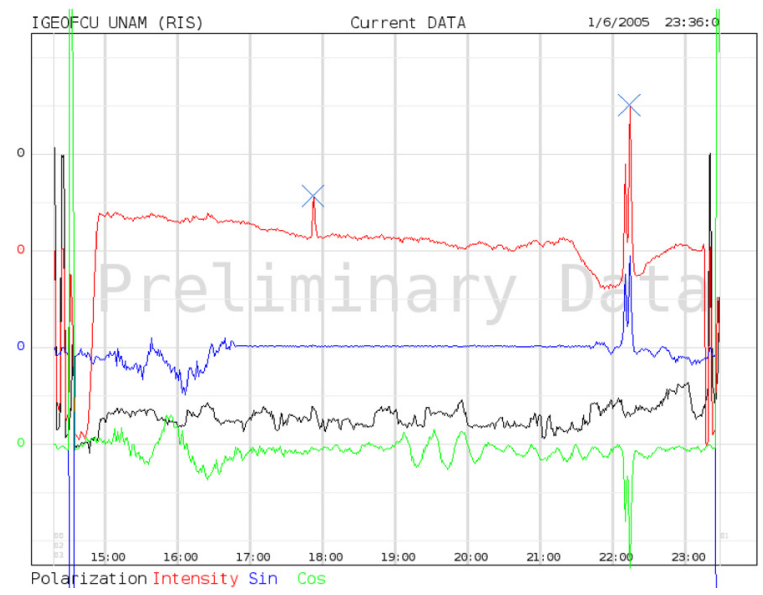

Figure 4 - Four channel plot published in internet in real time by xitris-www; Polarization vs. Time (black), the Intensity (red), the Sin, and the Cos components of the signal (blue and green respectively). The cross shows solar events detected by the RIS.

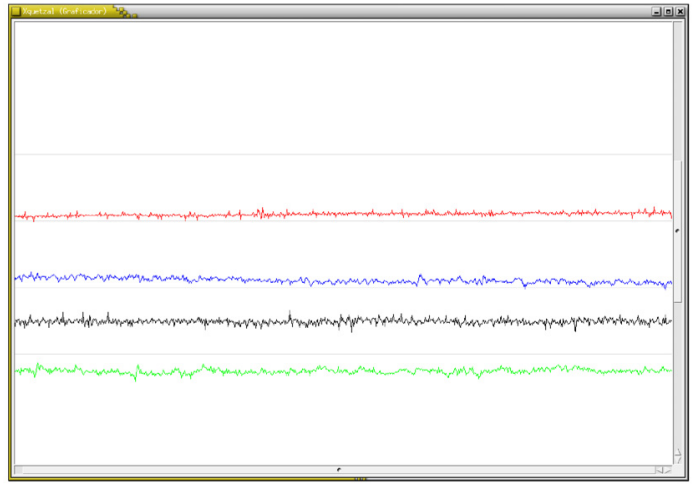

Figure 5 - Four channel plot from xitris-client (ollin interface) observed in the laboratory; this plot shows in real time the four signals from the radiotelescope.

Each interface can be running (and displayed) in different PCs. The information is carried trough the local network or internet inclusive, however the security for use public networks is not implemented yet.

The site of the RIS that use XITRIS can be founded in http://cintli.geofisica.unam.mx/

Until now, there are four implementations of xitris-www in different observatories:

- The RIS: The Solar Radio Interferometer in Mexico City, Mexico.

- The Mexart: The Mexican Array Telescope in Morelia, Mexico.

- The RT5: The Radio Telescope of 5 meters in Puebla, Mexico

- The IGA: The Institute of Geophysics in La Habana, Cuba.

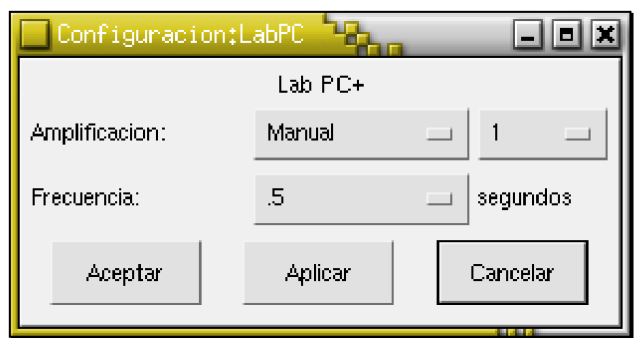

Figure 6 - Interface for the ADC configuration (tochtli interface); we can configure the amplification and the sample frequency of the LabPC+ ADC board in real time. The changes also affect the ollin plot window.

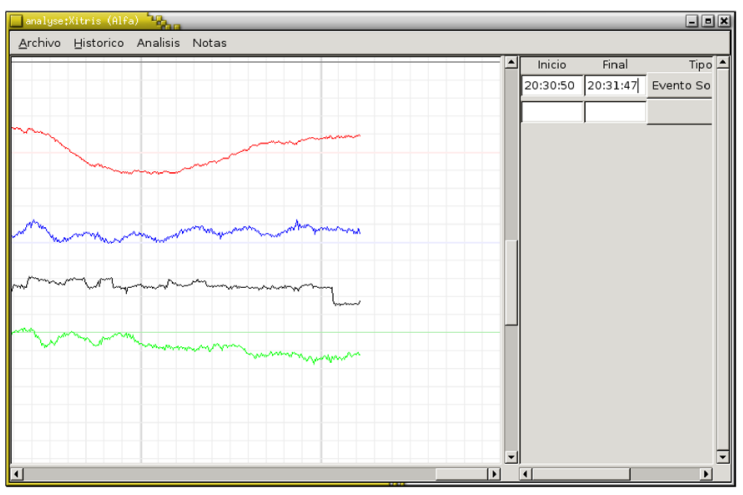

Figure 7 - Log of xitris (xitris-analyze); in this interface we can make annotations in real time over the signal, for example: in the case of malfunction of one component of the telescope, error in the pointing of the dish, no reference signal in the observation, the weather, a possible solar event, high noise, etc.

\section{CONCLUSIONS}

The Xitris code was first released in 2005, since then the code has been working without important interruptions in the RIS facilities. This shows that Xitris is very stable and reliable.

The distributed design of the code makes possible its adaptation to very restricted hardware infrastructure (old computers) and the $\mathrm{C}$ language is the key for a long life of the code.

On the way, we developed a useful library for the ADC LabPC+ board of National Instruments with a GNU/GPL License that works in the GNU/Linux environments with a high performance.

The eXtreme Programming was a very useful tool for dynamic design in a small groups of programmers.

We have installed the code in four facilities. Actually, Xitris is the responsible of deliver the data from the RIS to the Virtual Earth Solar Observatory (VESO) in Mexico.

Finally, Xitris can be expanded to incorporate new ADC boards and new environments that involves ADC boards on remote control. 


\section{Appendix A.1: CONVERSION BETWEEN 12 BITS DATA AND A2 32 BITS INTEGER IN C}

In particular, the Lab-PC+ output consists on two consecutive bytes (of 8 bits). Therefore, it is necessary to read two times the output register in order to get the 12 bits full data. The first read byte contains the 8 less significant bits, then the second read byte gives the 8 high significant bits (only use the last 4 bits). If the first bit of the second byte is zero, then the value is positive

$$
(010000000001)_{2}=2049_{10},
$$

if this bit is one, then the value is negative

$$
\begin{aligned}
(101111111111)_{2} & =(010000000000)_{2}+1_{2} \\
& =(010000000001)_{2}=-2049_{10} .
\end{aligned}
$$

In LabPC.c [2] we can found the implementation of the conversion between 12 bits data and $A 232$ bits integer in C:

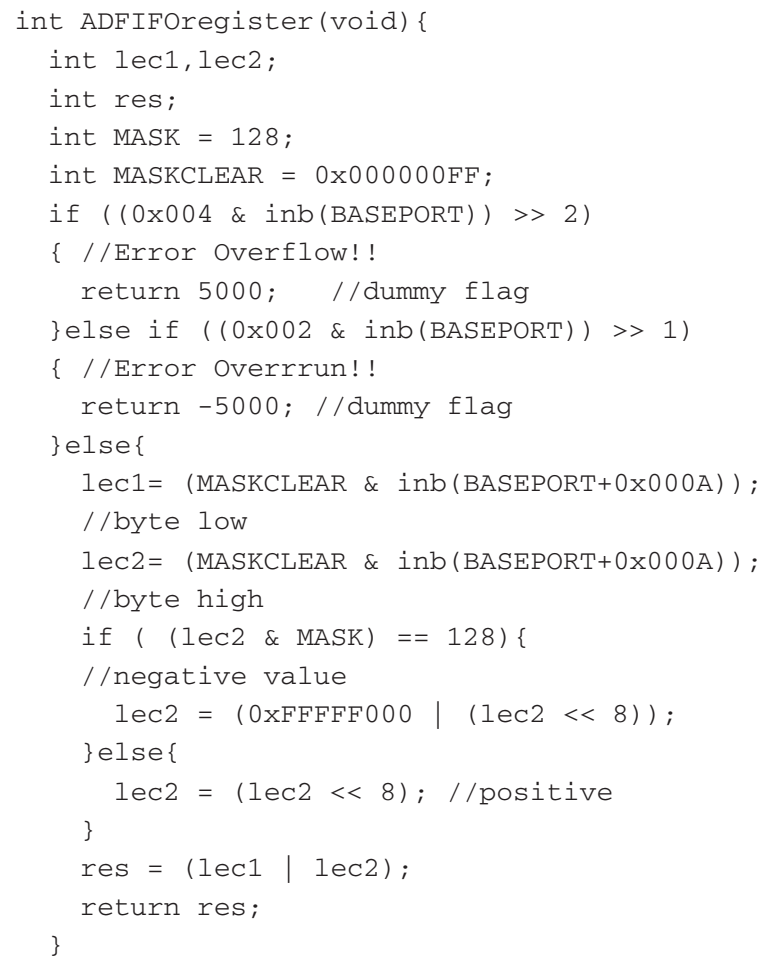

\section{APPENDIX A.2: SHARED MEMORY}

In the main.c code of Tochtli [2] is the definition to create the shared memory [3]:

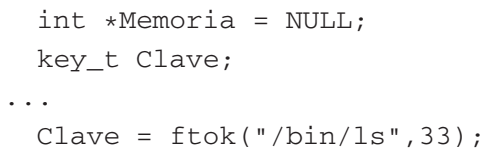

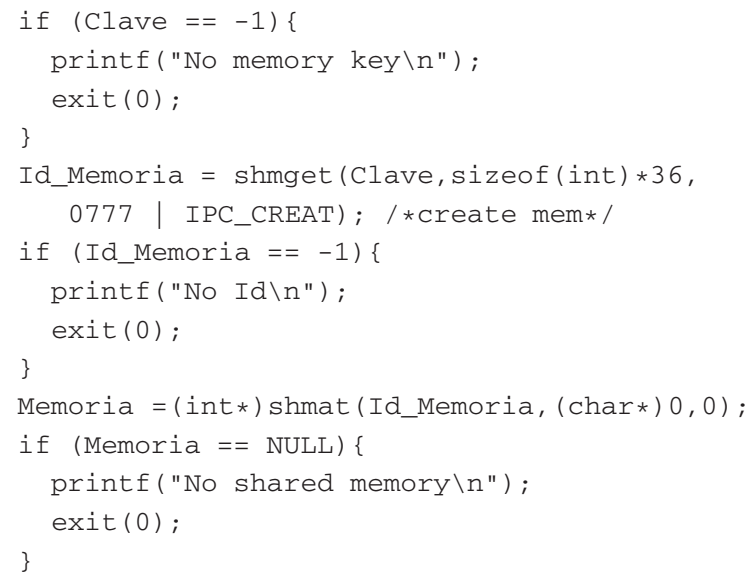

In the main.c code of Ollin [2] is the definition to read the shared memory [3]:

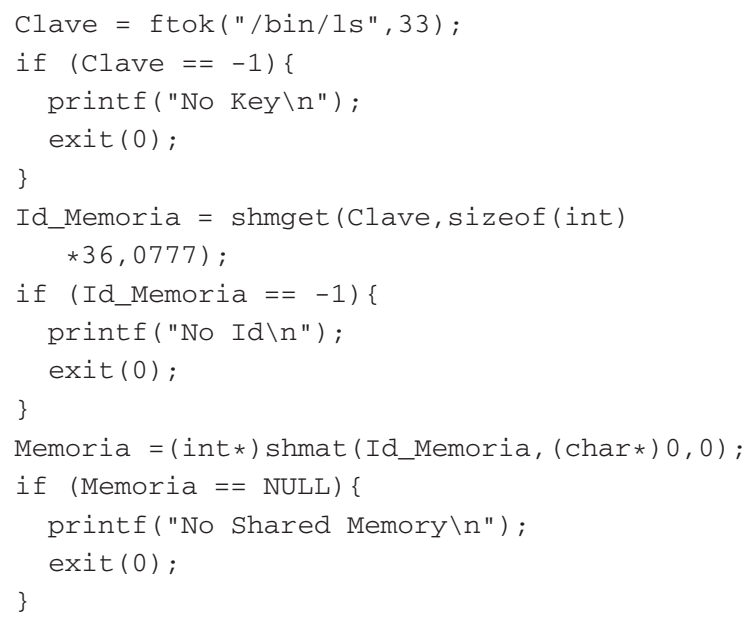

\section{ACKNOWLEDGMENTS}

The Debian user list to the invaluable help in several technical topics.

V. De la Luz thank Catedras-CONACyT project 1045.

\section{REFERENCES}

[1] BECK K. 2000. Extreme Programming Explained: Embrace Change. The XP Series. Addison-Wesley.

[2] DE LA LUZ V. 2014. Xquetzal xitris. http://sourceforge.net/projects/xquetzal/, April.

[3] GARCÍA FMM. 1993. Unix programación avanzada. Ra-ma.

[4] HALL M. 2000. Core servlets and JavaServer Pages. Prentice Hall PTR Core series. Prentice Hall PTR.

[5] HARLOW E. 1999. Developing Linux Applications with Gtk+ and Gdk. The Landmark Series. New Riders.

[6] KOCHAN SG \& WOOD PH. 1989. UNIX networking. Hayden Books UNIX system library. Hayden Books. 
[7] NISSANKE N. 1997. Realtime systems. Prentice Hall international series in computer science. Prentice Hall.

[8] TRAVIS J \& KRING J. 2007. LabView for Everyone: Graphical Programming Made Easy and Fun. National Instruments Virtual Instru- mentation Series. Prentice Hall.

[9] VAN WYK CJ. 1990. Data structures and C programs. AddisonWesley series in computer science. Addison-Wesley Pub. Co. 